\title{
BMJ Open HIV prevalence and factors associated with HIV infection among transgender women in Cambodia: results from a national Integrated Biological and Behavioral Survey
}

\author{
Srean Chhim, ${ }^{1}$ Chanrith Ngin, ${ }^{2}$ Pheak Chhoun, ${ }^{2}$ Sovannary Tuot, ${ }^{2}$ Cheaty Ly, ${ }^{3}$ \\ Phalkun Mun, ${ }^{4}$ Khondyla Pal, ${ }^{2}$ John Macom, ${ }^{5}$ Jean-Philippe Dousset, ${ }^{1}$ \\ Gitau Mburu, ${ }^{6}$ Siyan $\mathrm{Yi}^{2,7}$
}

To cite: Chhim S, Ngin C, Chhoun $\mathrm{P}$, et al. HIV prevalence and factors associated with HIV infection among transgender women in Cambodia: results from a national Integrated Biological and Behavioral Survey. BMJ Open 2017;7:e015390. doi:10.1136/ bmjopen-2016-015390

- Prepublication history and additional material are available. To view these files please visit the journal online (http://dx.doi. org/10.1136/bmjopen-2016015390.

Received 2 December 2016

Revised 18 May 2017

Accepted 18 May 2017

CrossMark

For numbered affiliations see end of article.

Correspondence to

Dr Siyan Yi; siyan@doctor.com

\section{ABSTRACT}

Objective To examine factors associated with HIV infection among transgender women in Cambodia.

Design Cross-sectional study.

Settings HIV high-burden sites including the capital city and 12 provinces.

Participants This study included 1375 sexually active transgender women with a mean age of 25.9 years (SD 7.1), recruited by using respondent-driven sampling for structured questionnaire interviews and rapid finger-prick HIV testing.

Primary outcome measure HIV infection detected by using Determine antibody test.

Results HIV prevalence among this population was $5.9 \%$. After adjustment for other covariates, participants living in urban areas were twice as likely to be HIV infected as those living in rural areas. Participants with primary education were 1.7 times as likely to be infected compared with those with high school education. HIV infection increased with age; compared with those aged 18-24 years, the odds of being HIV infected were twice as high among transgender women aged 25-34 years and 2.8 times higher among those aged $\geq 35$ years. Self-injection of gender affirming hormones was associated with a fourfold increase in the odds of HIV infection. A history of genital sores over the previous 12 months increased the odds of HIV infection by threefold. Transgender women with stronger feminine identity, dressing as a woman all the time, were twice as likely to be HIV infected compared with those who did not dress as a woman all the time. Having never used online services developed for transgender women in the past six months was also associated with higher odds of being HIV infected. Conclusions Transgender women in Cambodia are at high risk of HIV. To achieve the goal of eliminating HIV in Cambodia, effective combination prevention strategies addressing the above risk factors among transgender women should be strengthened.

\section{INTRODUCTION}

Globally, transgender women are at high risk for HIV infection, and little is known about the burden of HIV infection and its related
Strengths and limitations of this study

- This is a rare and the second ever Integrated Biological and Behavioral Survey among transgender women-one of the most vulnerable and understudied populations in Cambodia and globally.

- This study covered HIV high-burden sites including the capital city and 12 provinces, thus providing more nationally generalisable results.

- This study identified important risk factors for HIV infection among transgender women, which require being addressed in order to reduce HIV infection among this key population.

- Limitations of the study included potential bias of self-reported measures, participant sampling, the cross-sectional nature of the data that limits causation inferences and the backward selection of variables in multivariable analysis.

factors in this population. A 2013 systematic review showed that the global HIV prevalence among transgender women was $19 \%$, with low-income and middle-income countries having a slightly lower prevalence of $18 \%$ compared with $22 \%$ in high-income countries. ${ }^{1}$ The probability of being infected with HIV among transgender women was approximately 49 times higher than among the general adult population. ${ }^{1}$ Epidemiologically, transgender women share some HIV risk factors with other populations, such as ulcerative sexually transmitted infections (STIs), ${ }^{23}$ multiple substance use, victimisation, intimate partner violence, ${ }^{4}$ unprotected sex, ${ }^{5}$ multiple sex partners $^{6}$ and injecting drug use. ${ }^{5-7}$

However, transgender women encounter additional and unique biological, social, 
cultural, legal and economic issues, which increase their vulnerability to HIV. Transgender women are marginalised legally and economically through discriminative legislation and practice, which accelerates their vulnerability to HIV infection by confining their job options to sex work. Sex work in turn exacerbates their stigmatisation and alienation, ${ }^{8}$ and further increases their HIV risk. ${ }^{9}$ Stigmatisation and discrimination further increase the risk of HIV infection by preventing transgender women from seeking essential health information and services. ${ }^{11}$ Depression and low self-esteem, which are common psychological consequences of stigma, further weaken the bargaining power for condom use in sexual relationships, resulting in heightened vulnerability to HIV infection. ${ }^{512-15}$ Transgender women who have sex with men often engage in receptive anal intercourse, which increases their risk of HIV acquisition, compared with insertive sexual partners. ${ }^{2}$ Syndemics of illicit drug use, abuse by family members and mental health often occur together ${ }^{16}$ and are often associated with less likelihood of transgender women to use condoms during sex with commercial partners. ${ }^{17}$ In addition, the confluence of arbitrary arrests, ${ }^{18} 19$ violence, ${ }^{1620}$ economic pressures ${ }^{21}$ and other social problems further aggravates mental health risks among transgender women, causing them to engage in risky sexual behaviours. ${ }^{617}$

In Southeast Asia, a systematic review of studies from Indonesia, Thailand and Vietnam demonstrated that transgender women are particularly at high HIV risk, with an estimated HIV prevalence of $26.1 \%, 12.2 \%$ and $6.7 \%$, respectively. ${ }^{1}$ In Cambodia, up to 2012 , transgender women were traditionally included in HIV prevention programmes targeting men who have sex with men (MSM). As a result, their unique needs were not reflected in HIV programmes' goals. ${ }^{22}$ There was also a lack of transgender women-specific data to quantify the number reached by outreach programmes and services. In 2012, the first Integrated Biological and Behavioral Survey (IBBS) that specifically included transgender women in Cambodia found an HIV prevalence of $4.2 \%$ among this population, ${ }^{22}$ which was six times higher than the $0.7 \%$ prevalence among the general adult population aged $15-45$ years, ${ }^{23}$ and about twice higher than the $2.1 \%$ among MSM. ${ }^{24}$ This 2012 study also found that older age ( $>35$ years), residing in Siem Reap province (a major tourist site), having sex during or after drug use, not using a condom during the last anal sex and low self-esteem were associated with HIV infection among transgender women. ${ }^{22}$

Recognising the differences in HIV vulnerability and prevention needs between transgender women and MSM, in 2013 the National Center for HIV/AIDS, Dermatology and STD (NCHADS) separated these two populations in its revised Standard Operating Procedure known as Boosted Continuum of Prevention, Care and Treatment (B-CoPCT). ${ }^{25}$ Transgender women have since been considered a distinct at-risk group for HIV surveillance and programming. The National B-CoPCT approach aims to increase the uptake of HIV testing and counselling and other related services, ensure the quality of outreach and strengthen service delivery to meet specific needs of key populations, including transgender women, in-order to achieve Cambodia's 3.0 goal (ie, zero new HIV infection, zero discrimination and zero AIDS-related deaths) by 2020 .

This study was conducted to explore the HIV prevalence and identify risk factors associated with HIV infection among transgender women in Cambodia. The study findings will help determine ways in which services and policies can be tailored for this key population.

\section{METHODS}

\section{Study design and sites}

Between December 2015 and February 2016, a cross-sectional study was conducted in the capital city of Phnom Penh and 12 provinces, namely Battambang, Banteay Meanchey, Kampong Cham, Kandal, Koh Kong, Kampong Chhnang, Kampong Speu, Prey Veng, Preah Sihanouk, Siem Reap, Svay Rieng and Tbong Khmum. These sites were purposively selected from the 23 HIV high-burden operational districts identified by NCHADS. ${ }^{26} 27$ The study was a combination of a biological (blood test) and behavioural (face-to-face interview) survey using Respondent-Driven Sampling method to reach the target population.

\section{Sample size and sampling procedures}

The sample size calculation was based on an assumption that HIV prevalence would have changed between the first and the current IBBS. The minimum sample size required for this study was 1380 , which would result in sufficient power to detect a priori significant difference in HIV prevalence, as expressed in the following null and alternative hypotheses. The hypothesis test was that of one-sample proportion compared with the null hypothesis.

The null hypothesis $\left(\mathrm{H}_{0}\right)$ : HIV prevalence among transgender women remains constant at $4.2 \%$, similar to that found in the 2012 IBBS.

The alternative hypothesis $\left(\mathrm{H}_{\mathrm{a}}\right.$ : HIV prevalence among transgender women has decreased by $1.2 \%$ from $4.2 \%$ in 2012 to $3.0 \%$ in the present IBBS.

The sample size was calculated using the following formula and assumptions:

$$
n=\frac{p_{0} q_{0}\left(z_{1-\alpha}+z_{1}-\beta \sqrt{\frac{p_{1} q_{1}}{p_{0} q_{0}}}\right)^{2}}{\left(p_{1}-p_{0}\right)^{2}}
$$

- $\mathrm{P}_{0}=$ estimated proportion in $\mathrm{H}_{0}$ (In the most recent survey, HIV prevalence among transgender women was $4.2 \%$ in 2012).

- $\mathrm{P}_{1}=$ estimated proportion in $\mathrm{H}_{\mathrm{a}}$ (The expected HIV prevalence among transgender women in the current study is about $3.0 \%$ ).

- $\mathrm{Z}_{(1-\alpha)\rangle}=$ significance level at $5 \%$ in response to one-sided test $(Z$ score $=1.645)$. 
- $\mathrm{Z}(1-\beta)=$ power level of $80 \%(\mathrm{Z}$ score $=0.83)$.

- Refusal rate of $10 \%$.

- Design effect is assumed to be one.

This sample size was stratified by study site. Roughly half of the estimated transgender women in each study site were recruited. However, in five provinces where the estimated number of transgender women was $<100$, all transgender women were recruited. Potential participants were included in the study if they (1) were biologically male at birth and self-identified as a woman or third gender, (2) were Khmer-speaking, (3) were at least 18 years of age at the time of screening, (4) reported having had sex with at least one man within the past 12 months and (5) were able and willing to provide an informed consent.

Among the 13 study sites, data collection was conducted in 20 locations (6 locations in Phnom Penh and 14 locations in the remaining provinces). The number of the selected locations was determined based on the required sample size in each study site. Our participants, including the seeds and the people the seeds referred, had to meet the eligibility criteria. These participants were recruited from the entire transgender population in the study sites. However, the initial seeds at each site were recruited through our implementing partners. First, four 'seeds' (two seeds aged 18-24 and two seeds 25 or older) who were well connected with other transgender women in each location were selected by outreach workers from implementing partners (local non-governmental organisations (NGOs)) based in the selected locations. These seeds had to meet the above-mentioned eligibility criteria for participation and have an established and large social network consisting of $\geq 10$ other transgender women in their given location. Eligibility to participate as a seed was determined by the leader of the data collection team, using a paper-based eligibility form.

Second, each seed was given a personal identity number and enrolled in the study. Third, each seed was given three coupons and asked to refer three additional transgender women. US $\$ 2$ was given to each seed for a successful referral. Each seed was expected to extend to 3-6 'recruitment waves' in each location. If the initial seeds did not recruit participants, or if enrolment was halted because all recruitment chains had 'dried up' (ie, stopped recruiting), additional seeds were selected. In total, 80 seeds were selected by the outreach workers, and a total of 1375 transgender women were enrolled in the study. Referred participants were initially screened by the data collectors for eligibility.

\section{Data collection training}

Data were collected by three teams; each team comprised one field supervisor, five interviewers, one lab technician and one counsellor. Lab technicians and counsellors were from the Municipal or Provincial AIDS and STI Program of the study sites. Data collection teams were trained for 3 days on study protocol, research ethics, interview techniques and data collection procedures provided by the principal investigators and research coordinators.

\section{Data collection procedures}

Biological data collection

To determine HIV prevalence, all participants received onsite rapid finger-prick testing. Pre-test counselling was provided by qualified, well-trained counsellors working for voluntary confidential counselling and testing (VCCT) centres. Participants could receive their HIV test result verbally after the questionnaire interview. A blood sample was obtained from each participant by a trained laboratory technician through finger-prick and tested for HIV using Determine test, in keeping with the national protocol. ${ }^{28}$ Post-test counselling was provided for each participant regardless of their HIV test result by the same counsellor who conducted the pre-test counselling in accordance with NCHADS HIV testing guidelines. ${ }^{28}$ Participants who were HIV reactive and did not know their HIV status were referred by the counsellor for confirmatory testing at the nearest VCCT centre. The quality of HIV tests was monitored using quality control samples. Discordant results between the screening and confirmatory tests were investigated to elucidate potential causes and minimise potential erroneous results. In the case of human error, individuals performing screening tests were retrained before resuming their involvement in the study.

After HIV testing, the participant was interviewed by a well-trained interviewer using a computer-assisted survey instrument. The questionnaire was set up in Qualtrics, a web-based application, and run on an Android tablet. HIV testing and interviews were conducted in the selected 20 locations including drop-in centres, private houses and offices of implementing partners, depending on participants' convenience.

\section{Questionnaire development and measures}

The questionnaire was developed using standardised and validated tools adapted from TGIBBS-2012, ${ }^{22}$ FHI 360 's guideline for Behavioral Surveillance Survey ${ }^{29}$ and NCHADS's Boosted-CoPCT ${ }^{25}$ to measure key variables related to the objectives of the study. The questionnaire was initially drafted in English and translated into Khmer, the national language of Cambodia. Consultative meetings to improve the draft questionnaire were held with representatives of transgender women, communities and NGOs working with transgender women, as well as researchers and practitioners working on HIV and AIDS in Cambodia. The questionnaire was pre-tested with 20 transgender women in Phnom Penh and finalised based on findings.

The questionnaire collected information on socioeconomic characteristics, transgender identity and related experiences, sexual behaviours and condom use with different types of sexual partners, HIV/STI screening and care seeking behaviours, substance use and exposure to HIV programmes. For details of the questionnaire, please see online supplementary file 1 . 


\section{Data analyses}

HIV prevalence was calculated by dividing the total number of participants with HIV reactive test results with the total number of participants. To examine the associated factors of HIV infection, we conducted both bivariate and multivariable analyses. In the bivariate analyses, we compared all characteristics and behavioural variables of participants with a reactive test result to those of participants with a non-reactive test result. Among participants who tested HIV positive, additional analyses were conducted to assess whether there was a significant difference in sexual behaviours of those who were aware of their HIV-positive status and of those who were not. $\chi^{2}$ test or Fisher's exact test (for an expected cell value of $\leq 5$ ) was used for categorical variables, while Student's t-test was used for continuous variables. To facilitate the model, some continuous variables, such as age, were transformed to categorical variables. A multivariable logistic regression model was constructed to examine independent factors associated with HIV infection. Variables with a significance level of $p<0.05$ in the bivariate analyses were simultaneously included in the model. Backward elimination method was then used to eliminate variables with the highest p-value one-by-one from the model. STATA V.12.0 for Windows was used to conduct the data analyses.

\section{ETHICAL STATEMENT}

Participation in this study was voluntary, and a written informed consent was obtained from each study participant after a detailed description of the study objective and procedures was explained to them. Participants were informed that they could stop responding to questions and discontinue their participation at any time. Interviews were conducted at a private place, and confidentiality was enhanced by assigning a unique and anonymous code to each participant. No personal identifiers were contained in the questionnaires or dataset.

\section{RESULTS}

\section{HIV prevalence}

Of 1375 participants tested, $81(5.9 \%)$ had an HIV reactive test; of whom, $42(52 \%)$ were not aware of their HIV status prior to the study. Out of the 39 cases who already knew their status, $37(94.9 \%)$ were currently on HIV treatment, with the other two reporting having dropped out of the treatment.

\section{Socio-demographic characteristics}

As shown in table 1 , the majority of the participants $(83.4 \%)$ were recruited from urban communities; $53.0 \%$ were younger than 25 years and $97.2 \%$ had never been married. More than two-thirds of participants (68.6\%) had completed high school and $9.1 \%$ had a higher education. The most common main occupations reported by the participants were hair dressers/beauticians (35.1\%), labourers/farmers $(17.5 \%)$ and entertainment workers
(14.8\%). More than one-third of participants (38.6\%) reported an average monthly income in the past six months of US $\$ 100-199$, while $16.5 \%$ reported it to be $>$ US $\$ 300$. Regarding gender identity, $42.2 \%$ identified themselves as female, while $57.2 \%$ identified themselves as third gender. Almost half $(48.0 \%)$ of the participants reported dressing as a woman all the time; $45 \%$ ever used hormone/non-hormone substance; and $14.0 \%$ ever injected hormones.

HIV prevalence was significantly higher among participants living in urban communities compared with participants living in rural communities $(6.5 \%$ vs $2.6 \%$, $\mathrm{p}=0.02$ ). The HIV prevalence was also significantly different by age group: $3.0 \%$ of participants in the age group of $18-24$ years, $8.2 \%$ in $25-34$ years, $13.1 \%$ in $35-44$ years and $11.4 \%$ in 45 years or older $(p<0.001)$. HIV prevalence was also significantly higher among those who never attended school or dropped out of primary school compared with those who at least attended high school or higher $(10.4 \%$ vs $8.0 \%, \mathrm{p}<0.001)$. In addition, HIV prevalence was significantly higher among transgender women who were NGO staff (mainly HIV-focused NGOs) $(20.6 \%)$ and those who were unemployed (9.4\%) $(\mathrm{p}=0.03)$. HIV prevalence was significantly higher among transgender women who reported dressing as women all the time $(8.5 \%$ vs $3.5 \%, \mathrm{p}=0.001)$, among those who ever used female hormones $(8.1 \%$ vs $4.1 \%, \mathrm{p}=0.02)$ and among those who ever injected hormones $(9.1 \%$ vs $5.2 \%$, $\mathrm{p}=0.002$ ). Moreover, HIV prevalence was significantly higher among transgender women who reported never using online services developed specifically for MSM or transgender women, such as Facebook group pages or various websites $(9.5 \%$ vs $3.9 \%, \mathrm{p}<0.001)$.

\section{Sexual behaviours}

Sexual behaviours among transgender women with reactive and non-reactive HIV test are shown in table 2. An overwhelming majority of study participants $(87.5 \%)$ reported only having had receptive anal sex in the past 12 months. Of the total, $86.0 \%$ reported having had anal sex with at least one man within the previous three months, with the median number of male sex partners in the past three months being three (IQR 1-9). Of those who were sexually active with men in the past three months, $61.9 \%$ reported having used a condom at last sex with a man.

A total of $94.8 \%$ of participants who had anal sex in the prior three months reported having at least one non-transactional male sex partner within the past three months; of which, $62.1 \%$ reported always using condoms with their non-transactional male sex partners. Of participants who had sex in the past three months, $41.8 \%$ reported having sex with at least one man in exchange for money or gift; of whom $60.0 \%$ reported always using condoms with the partners.

Table 2 also shows that HIV prevalence was significantly higher among participants who reported having had anal sex with a man in the past three months $(6.5 \%$ vs $2.1 \%$, $\mathrm{p}=0.02$ ) and among those who reported having had sex 
Table 1 Comparison of socio-demographic, gender identity and hormone use characteristics of transgender women with and without a reactive HIV test

\begin{tabular}{|c|c|c|c|c|}
\hline \multirow{3}{*}{$\begin{array}{l}\text { Socio-demographics, gender } \\
\text { identity and hormone use }\end{array}$} & \multirow{3}{*}{$\begin{array}{l}\text { Total }(n=1375) \\
n(\%)\end{array}$} & \multicolumn{3}{|l|}{ HIV test result } \\
\hline & & \multirow{2}{*}{$\begin{array}{l}\text { Reactive }(\mathrm{n}=81) \\
\mathrm{n}(\%)\end{array}$} & \multicolumn{2}{|c|}{ Non-reactive $(n=1294)$} \\
\hline & & & n (\%) & p Value* \\
\hline \multicolumn{5}{|l|}{ Community type } \\
\hline Urban & $1146(83.4)$ & $75(6.5)$ & $1071(93.5)$ & 0.02 \\
\hline Rural & $229(16.6)$ & $6(2.6)$ & $223(97.4)$ & \\
\hline \multicolumn{5}{|l|}{ Age (years) } \\
\hline $18-24$ & $729(53.0)$ & $22(3.0)$ & $707(97.0)$ & $<0.001$ \\
\hline $25-34$ & $503(36.6)$ & $41(8.2)$ & $462(91.8)$ & \\
\hline$\geq 35$ & $143(10.4)$ & $18(12.6)$ & $125(87.4)$ & \\
\hline \multicolumn{5}{|l|}{ Current marital status } \\
\hline Married & $7(0.5)$ & $0(0.0)$ & $7(100)$ & 0.47 \\
\hline Widowed/divorced/separated & $18(1.3)$ & $1(5.6)$ & $17(94.4)$ & \\
\hline Never married & $1334(97.2)$ & $78(5.9)$ & $1256(94.1)$ & \\
\hline Refused to answer & $16(1.2)$ & $2(15.4)$ & $11(84.6)$ & \\
\hline \multicolumn{5}{|l|}{ Years of formal education completed } \\
\hline Primary (0-6years) & 307 (22.3) & $32(10.4)$ & $275(89.6)$ & $<0.001$ \\
\hline High school or higher (>7 years) & $1068(77.7)$ & $49(4.6)$ & $1019(95.4)$ & \\
\hline \multicolumn{5}{|l|}{ Main occupation } \\
\hline Unemployed & $64(4.7)$ & $6(9.4)$ & $58(90.6)$ & 0.03 \\
\hline Hair dresser/beautician & $482(35.1)$ & $24(5.0)$ & $458(95.0)$ & \\
\hline Office worker & $50(3.6)$ & $3(6.0)$ & $47(94.0)$ & \\
\hline Labour/farmer & $241(17.5)$ & $15(6.2)$ & $226(93.8)$ & \\
\hline Seller & $149(10.8)$ & $10(6.7)$ & $139(93.3)$ & \\
\hline Entertainment worker & $203(14.8)$ & $10(4.9)$ & $193(95.1)$ & \\
\hline Student & $108(7.9)$ & $1(0.9)$ & $107(99.1)$ & \\
\hline $\begin{array}{l}\text { Non-governmental organisation } \\
\text { staff }\end{array}$ & $34(2.5)$ & $7(20.6)$ & $27(79.4)$ & \\
\hline Other & $44(3.2)$ & $5(11.4)$ & $39(88.6)$ & \\
\hline \multicolumn{5}{|c|}{ Monthly income in the past six months (US\$) } \\
\hline$<100$ & $351(25.6)$ & $22(6.3)$ & $329(93.7)$ & 0.70 \\
\hline $100-199$ & $530(38.6)$ & $29(5.5)$ & $501(94.5)$ & \\
\hline 200-299 & 266 (19.3) & $19(7.1)$ & $247(92.9)$ & \\
\hline$\geq 300$ & $226(16.5)$ & $11(4.9)$ & $215(95.1)$ & \\
\hline \multicolumn{5}{|l|}{ Gender identity (self-identified) } \\
\hline Female & $580(42.2)$ & $29(5.0)$ & $551(95.0)$ & 0.35 \\
\hline Third gender & $786(57.2)$ & $52(6.6)$ & $734(93.4)$ & \\
\hline Uncertain & $8(0.6)$ & $0(0.0)$ & $8(100)$ & \\
\hline \multicolumn{5}{|l|}{ Frequency of dressing as a woman } \\
\hline All the time & $660(48.0)$ & $56(8.5)$ & $604(91.5)$ & 0.001 \\
\hline Not all the time & $715(52.0)$ & $25(3.5)$ & $689(96.5)$ & \\
\hline \multicolumn{5}{|l|}{ Ever injected hormones } \\
\hline No & $1123(81.7)$ & $58(5.2)$ & $1065(94.8)$ & 0.02 \\
\hline Yes & 252 (18.3) & $23(9.1)$ & $229(90.9)$ & \\
\hline
\end{tabular}


Table 1 Continued

\begin{tabular}{|c|c|c|c|c|}
\hline \multirow{3}{*}{$\begin{array}{l}\text { Socio-demographics, gender } \\
\text { identity and hormone use }\end{array}$} & \multirow{3}{*}{$\begin{array}{l}\text { Total }(n=1375) \\
\mathrm{n}(\%)\end{array}$} & \multicolumn{3}{|l|}{ HIV test result } \\
\hline & & \multirow{2}{*}{$\begin{array}{l}\text { Reactive ( } \mathrm{n}=81) \\
\mathrm{n}(\%) \\
\end{array}$} & \multicolumn{2}{|c|}{ Non-reactive $(n=1294)$} \\
\hline & & & n (\%) & p Value* \\
\hline No & $1358(98.8)$ & $77(5.7)$ & $1281(94.3)$ & 0.002 \\
\hline Yes & $17(1.2)$ & $4(25.5)$ & $13(76.5)$ & \\
\hline \multicolumn{5}{|c|}{ Ever shared needles when injecting hormones/beauty substances } \\
\hline No & $1355(98.5)$ & $78(5.8)$ & $550(94.2)$ & 0.33 \\
\hline Yes & $20(3.2)$ & $2(10.0)$ & $18(90.0)$ & \\
\hline \multicolumn{5}{|c|}{ Ever used online services developed for men who have sex with men/transgender (eg, Facebook, website) } \\
\hline No & $483(35.1)$ & $46(9.5)$ & $437(90.5)$ & $<0.001$ \\
\hline Yes & 892 (64.9) & $35(3.9)$ & 857 (96.1) & \\
\hline
\end{tabular}

${ }^{*} \mathrm{X}^{2}$ or Fisher's exact test was used as appropriate.

with at least one man in exchange for money or gift in the prior three months $(8.1 \%$ vs $5.4 \%, \mathrm{p}=0.04)$ compared with participants who did not report these sexual behaviours.

\section{Sexual behaviours and awareness of HIV status}

Among the participants who had HIV reactive tests, additional analyses were conducted to see if there were differences in sexual behaviours among those who self-reported a positive status and those who did not. Participants who did not report or were unaware of their positive status were significantly less likely to report using a condom at last sexual intercourse $(52.4 \%$ vs $79.0 \%$, $\mathrm{p}=0.01$ ). No other significant differences were found.

\section{Sexually transmitted infections}

Overall, $14.0 \%$ of participants reported having had at least one STI symptom in the past 12 months. Anal and perianal symptoms were most commonly reported $(6.1 \%)$. As shown in table 3, HIV prevalence was significantly higher among participants who reported having had an STI symptom compared with that among participants who did not have an STI symptom in the past 12 months ( $11.4 \%$ vs $5.0 \%, \mathrm{p}<0.001)$. HIV prevalence was also significantly higher among participants who reported having had an ulceration or sore in the genital area in the past 12 months compared with that among participants who did not have it $(15.2 \%$ vs $5.6 \%, \mathrm{p}<0.001)$.

\section{Substance use}

As shown in table 5, the majority $(75.9 \%)$ of participants reported drinking at least one can of beer or a glass of wine in the past three months, while $10.1 \%$ reported using amphetamine-type stimulants (Yama, Crystal Ice, Ecstasy) and $0.9 \%$ reported using other drugs (marijuana, heroin) in the previous 12 months. Of total, $1.5 \%$ reported having injected any illicit drugs in the past three months.

Table 4 also shows that HIV prevalence was significantly higher among participants who did not drink alcohol when compared with that among participants who reported drinking at least one can of beer or a glass of wine in the past three months $(8.5 \%$ vs $5.1 \%, \mathrm{p}=0.02)$.
Moreover, HIV prevalence was significantly higher among participants who reported injecting any illicit drugs in the past threemonths compared with participants who did not $(15.0 \%$ vs $5.8 \%, \mathrm{p}=0.01)$.

\section{Factors associated with HIV infection}

Table 5 presents independent factors associated with HIV infection in multivariable logistic regression analyses. After adjustment for other covariates, participants living in urban areas were twice as likely to be HIV infected as those living in rural areas (adjusted OR (AOR) 2.7, 95\% CI 1.1 to 6.5 ). Participants with primary education were 1.7 times as likely to be infected compared with those with high school education (AOR 1.7, 95\% CI 1.0 to 2.9). HIV infection increased with age; compared with those aged 18-24 years, the odds of being HIV infected were twice as high among transgender women aged 25-34 years (AOR 2.1, 95\% CI 1.2 to 3.6) and 2.8 times higher among those aged $\geq 35$ years (AOR 2.8, 95\% CI 1.3 to 6.1 ). Self-injection of gender affirming hormones was associated with a fourfold increase in the odds of HIV infection (AOR 4.4, 95\% CI 1.1 to 17.3). A history of genital sores over the previous 12 months increased the odds of HIV infection by threefold (AOR 3.0, 95\% CI 1.2 to 7.8 ). Transgender women with stronger feminine identity, dressing as a woman all the time, were twice as likely to be HIV infected compared with those who did not dress as a woman all the time (AOR 2.1, 95\% CI 1.2 to 3.8). Having never used online services developed for transgender women in the past six months was also associated with higher odds of being HIV infected (AOR 1.9, 95\% CI 1.2 to 3.2).

\section{DISCUSSION}

This study reveals an HIV prevalence of 5.9\% among transgender women in Cambodia. This prevalence was about 20 times higher than the $0.3 \%$ among women attending antenatal care clinics in 2014 who represent the general adult population aged $15-45$ years, ${ }^{30}$ and about 2.5 times higher than the $2.3 \%$ among MSM in $2014 .{ }^{26}$ 
Table 2 Comparisons of sexual behaviours among transgender women with reactive and non-reactive HIV test

\begin{tabular}{|c|c|c|c|c|}
\hline \multirow[b]{3}{*}{ Sexual behaviours } & \multirow{3}{*}{$\begin{array}{l}\text { Total }(n=1375) \\
n(\%)\end{array}$} & \multicolumn{3}{|l|}{ HIV test result } \\
\hline & & \multirow{2}{*}{$\begin{array}{l}\text { Reactive }(\mathrm{n}=81) \\
\mathrm{n}(\%)\end{array}$} & \multicolumn{2}{|c|}{ Non-reactive $(n=1294)$} \\
\hline & & & $\mathrm{n}(\%)$ & p Value* \\
\hline \multicolumn{5}{|c|}{ Role in anal sex with a man (past 12 months) } \\
\hline Insertive & $29(2.2)$ & $1(3.5)$ & $28(96.5)$ & 0.73 \\
\hline Receptive & $1145(87.5)$ & $72(6.3)$ & $1073(93.7)$ & \\
\hline Both & 135 (10.3) & $7(5.2)$ & $128(94.8)$ & \\
\hline \multicolumn{5}{|c|}{ Had anal sex with a man (past three months) } \\
\hline No & $192(14.0)$ & $4(2.1)$ & $188(97.9)$ & 0.02 \\
\hline Yes & $1183(86.0)$ & $77(6.5)$ & $1106(93.5)$ & \\
\hline \multicolumn{5}{|c|}{ Number of male sexual partners (past three months) } \\
\hline$<2$ & $512(37.3)$ & $24(4.7)$ & $488(95.3)$ & 0.14 \\
\hline$\geq 2$ & $861(62.7)$ & $57(6.6)$ & $804(93.4)$ & \\
\hline \multicolumn{5}{|c|}{ Used a condom at last sex (past three months) } \\
\hline No & $451(38.1)$ & $27(6.0)$ & $423(94.0)$ & 0.57 \\
\hline Yes & $732(61.9)$ & $50(6.8)$ & $682(93.2)$ & \\
\hline \multicolumn{5}{|c|}{ Had anal sex with a man, not in exchange for money or gift (past three months) } \\
\hline No & $61(5.2)$ & $5(8.2)$ & $56(91.8)$ & 0.37 \\
\hline Yes & $1122(94.8)$ & $72(6.4)$ & $1050(93.6)$ & \\
\hline \multicolumn{5}{|c|}{ Number of male sexual partners, not in exchange for money or gift (past three months) } \\
\hline$<2$ & $357(31.9)$ & $24(6.7)$ & $333(93.3)$ & 0.78 \\
\hline$\geq 2$ & $763(68.1)$ & $48(6.3)$ & $715(93.7)$ & \\
\hline \multicolumn{5}{|c|}{ Condom use with male sexual partner not in exchange for money or gift (past three months) } \\
\hline Not always & $697(62.1)$ & $50(7.2)$ & $647(92.8)$ & 0.19 \\
\hline Always & $425(37.9)$ & $22(5.2)$ & $403(94.8)$ & \\
\hline \multicolumn{5}{|c|}{ Had anal sex with a man in exchange for money or gift (past three months) } \\
\hline No & $688(58.2)$ & $37(5.4)$ & $651(94.6)$ & 0.04 \\
\hline Yes & $495(41.8)$ & $40(8.1)$ & $455(91.9)$ & \\
\hline \multicolumn{5}{|c|}{ Number of male sexual partners in exchange for money or gift (past three months) } \\
\hline$<2$ & $161(32.5)$ & $12(7.5)$ & $149(92.6)$ & 0.72 \\
\hline$\geq 2$ & $334(67.5)$ & $28(8.4)$ & $306(91.6)$ & \\
\hline \multicolumn{5}{|c|}{ Condom use with male sexual partner in exchange for money or gift (past three months) } \\
\hline Not always & $164(40.0)$ & $12(7.3)$ & 152 (92.7) & 0.56 \\
\hline Always & $246(60.0)$ & $22(8.9)$ & $224(91.1)$ & \\
\hline
\end{tabular}

${ }^{*} \chi^{2}$ test or Fisher's exact test was used as appropriate.

This observed prevalence emphasises that transgender women in Cambodia are at high risk of HIV and is consistent with global literature regarding the high burden of HIV among this transgender population. ${ }^{1}$

Although statistically non-significant $(p=0.13)$, the prevalence found in this study was higher than the prevalence of $4.2 \%$ reported in the smaller $(\mathrm{n}=891)$ TGIBBS conducted in $2012^{22}$ and was therefore contrary to our hypothesised expectations of reduction in HIV prevalence among this population. Recent progress in Cambodia, where $73 \%$ of all estimated HIV-positive people or $93 \%$ of those who have been aware of their positive status are in care, ${ }^{31}$ had prompted us to hypothesise the lower prevalence. Although we have no concrete evidence regarding the non-significant increase of HIV prevalence, we suggest that it may be related to the sampling variation in the two surveys. The previous study recruited participants only from the capital city and five provinces (Phnom Penh, Battambang, Banteay Meanchey, Kampong Cham, Siem Reap and Preah Sihanouk), ${ }^{22}$ whereas our study expanded to additional eight provinces.

More than half (52\%) of those who had HIV reactive tests reported that they had never learnt about their HIV-positive status before they participated in the study. We believe that this self-reporting of the HIV status is realistic. Since we were concerned that those who were already aware of 
Table 3 Comparisons of sexually transmitted infection symptoms among transgender women with reactive and non-reactive HIV test

\begin{tabular}{|c|c|c|c|c|}
\hline \multirow[b]{3}{*}{ STI symptoms } & \multirow{3}{*}{$\begin{array}{l}\text { Total }(n=1375) \\
n(\%)\end{array}$} & \multicolumn{3}{|l|}{ HIV test result } \\
\hline & & \multirow{2}{*}{$\begin{array}{l}\text { Reactive (n=81) } \\
\mathrm{n}(\%)\end{array}$} & \multicolumn{2}{|c|}{ Non-reactive $(n=1294)$} \\
\hline & & & n (\%) & p Value* \\
\hline \multicolumn{5}{|c|}{ Had experienced any STI symptom (past 12 months) } \\
\hline No & $1182(86.0)$ & $59(5.0)$ & $1123(95.0)$ & $<0.001$ \\
\hline Yes & $193(14.0)$ & $22(11.4)$ & $171(88.6)$ & \\
\hline \multicolumn{5}{|c|}{ Ulceration or sores around the genitals (past 12 months) } \\
\hline No & $1329(96.7)$ & $74(5.6)$ & $1255(94.4)$ & $<0.001$ \\
\hline Yes & $46(3.4)$ & $7(15.2)$ & $39(84.8)$ & \\
\hline \multicolumn{5}{|c|}{ Swelling around the genitals (past 12 months) } \\
\hline No & $1359(98.8)$ & $80(5.9)$ & $1279(94.1)$ & 1.000 \\
\hline Yes & $16(1.2)$ & $1(6.3)$ & $15(93.7)$ & \\
\hline \multicolumn{5}{|c|}{ Abnormal urethral discharge (past 12 months) } \\
\hline No & $1339(97.4)$ & $77(5.8)$ & $1262(94.2)$ & 0.16 \\
\hline Yes & $36(2.6)$ & $4(11.1)$ & $32(88.9)$ & \\
\hline \multicolumn{5}{|c|}{ STI symptoms around the anus (past 12 months) } \\
\hline No & $1291(93.9)$ & $73(5.6)$ & $1218(94.4)$ & 0.15 \\
\hline Yes & $84(6.1)$ & $8(9.52)$ & $76(90.48)$ & \\
\hline \multicolumn{5}{|c|}{ STI symptoms in the mouth or throat (past 12 months) } \\
\hline No & $1336(97.2)$ & $76(5.7)$ & $1260(94.3)$ & 0.07 \\
\hline Yes & $39(2.8)$ & $5(12.82)$ & $34(87.18)$ & \\
\hline
\end{tabular}

${ }^{*} \chi^{2}$ or Fisher's exact test was used as appropriate.

their HIV status may be unwilling to report their status, we decided to use well-trained counsellors who all were from the government's VCCT centres. These counsellors had many years of experience in providing VCCT services and were better equipped to cope with difficult situations. In Cambodia, we believe people are more likely to trust their counsellors, as opposed to interviewers. However, as in all self-reported measures, the potential for response bias cannot be entirely ruled out.

This study also reports important findings related to factors associated with HIV infection among transgender women in Cambodia. First, the HIV prevalence among transgender women residing in urban communities was twice as high compared with that among transgender women living in rural communities. This finding is similar to that in a previous study that found a higher HIV prevalence in transgender women living in urban areas of Siem Reap and Phnom Penh compared with that among transgender women living in other provinces. ${ }^{22}$ Findings from our study suggest that these differences may not necessarily be attributable to common sexual risk practices. The mean number of sexual partners ( 8.4 vs $7.9, p=0.70$ ) and the mean number of male commercial sexual partners ( 0.7 vs $0.7, \mathrm{p}=0.48)$ in the past three months among transgender women living in urban and rural communities were not significantly different. In addition, the rates of inconsistent condom use with male non-commercial sexual partners in the past three months were also similar in the two groups $(58.0 \%$ vs $62 \%, \mathrm{p}=0.32)$.

Given the failure of sexual risk behaviours to sufficiently account for the difference in HIV prevalence, these data suggest that a higher HIV prevalence among sexual partners of transgender women in urban communities may possibly explain the higher probability of urban transgender women to get infected, although this may not be stated with certainty. This is particularly relevant given that previous studies among all transgender women found a higher prevalence of HIV in urban areas. ${ }^{22}$ Studies among MSM in Cambodia have also found a higher prevalence of HIV in urban areas. ${ }^{24}{ }^{32}$ In addition, a recent national sentinel survey among women attending antenatal care clinics found that HIV prevalence among women in urban areas was consistently high, ${ }^{30}$ implying that HIV prevalence in urban areas among most populations, who potentially include transgender women and their partners, is high. It is also possible that transgender population in urban communities face unique or additional risks compared with other members of this population in rural areas, ${ }^{22}$ which could also contribute to the difference in HIV prevalence between urban and rural communities. Still, it is possible that those who knew they were positive may have moved to urban centres for care and treatment, which unfortunately cannot be examined further in this 
Table 4 Comparisons of substance use among transgender women with reactive and non-reactive HIV test

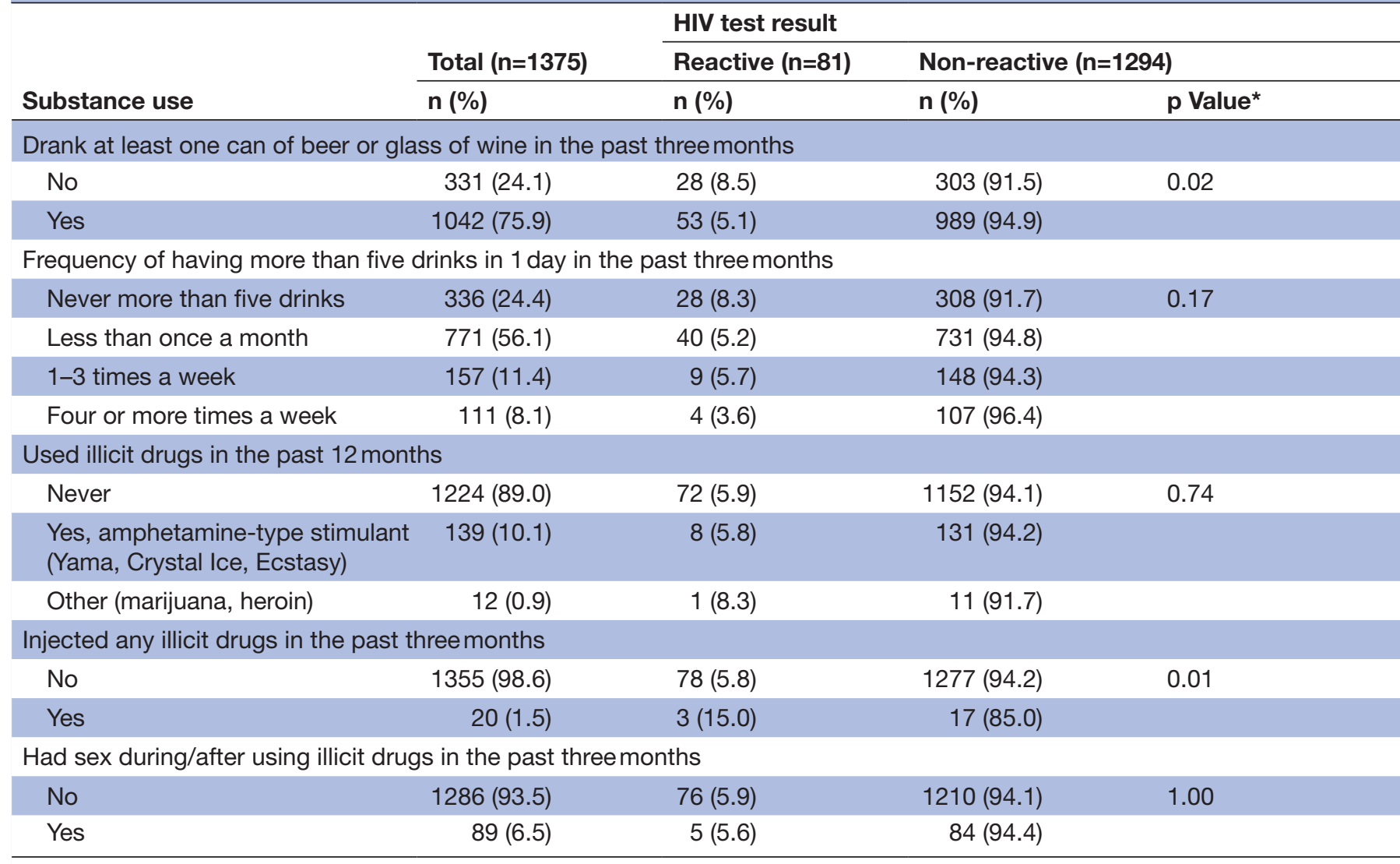

${ }^{*} \chi^{2}$ or Fisher's exact test was used as appropriate.

cross-sectional study. Further research is needed with regard to this observation.

Second, HIV prevalence among older transgender women ( $\geq 25$ years) was significantly higher compared with that among their younger counterparts. This finding is similar to those of previous studies in Cambodia ${ }^{22}$ and Thailand. ${ }^{3}$ A possible explanation is that older transgender women had been exposed to risks for a longer period of time; the number of their sexual partners accumulated over time and thus increased cumulative probability of HIV acquisition. ${ }^{22}$

Third, our study suggests that more overt feminine expression (eg, those who dressed as a woman all the time) was associated with a higher HIV prevalence. A study in the USA found that transgender women who disclosed their female gender identity possessed a higher HIV prevalence. ${ }^{33}$ In our study, these transgender women who dressed as a woman all the time had higher numbers of both male non-commercial (mean $=8.1$ vs 5.1, $\mathrm{p}=0.004$ ) and male commercial sexual partners (mean $=3.7 \mathrm{vs} 1.6$, $\mathrm{p}<0.001$ ), compared with those who did not. Making their female status visible could render more chances to meet with men. However, it could make them difficult to find a decent job due to stigmatisation and discrimination, thus leading them to engage in sex work. This finding is in line with existing literature showing that transgender women try to have sex with men-both casual and paid sex-to prove or validate their female gender identity. ${ }^{34-36}$ In these contexts, 'sex work provides both desired gender affirmation and economic stability, often with greater financial rewards for sex without a condom' ${ }^{34}$ The desire to affirm their gender identity and attractiveness to men also incentivises them to engage in casual sex with multiple partners. ${ }^{15}$ This sexual behaviour may make them more prone to HIV infection.

There is a tendency to self-inject gender-affirming hormones, often with shared needles, among transgender women in Cambodia. ${ }^{37}$ This risky practice was associated with an increased risk of HIV infection, implying that self-injecting was performed through unsafe means, and that injecting by a trained health or other professional might mitigate the likelihood of HIV infection. Although hormone injection to augment femininity is becoming more common, it can potentially cause adverse health effects among transgender women. ${ }^{338}$ A Thai study also found that transgender women who injected hormone to make them more feminine had a higher HIV prevalence. ${ }^{3}$

Fourth, transgender women who completed or dropped out of primary school had a higher HIV prevalence than those with high school education. This finding confirms results of the TGIBBS 2012 in Cambodia ${ }^{22}$ and other studies $^{639}$ that associated low education with high HIV prevalence among transgender women. This has important implications for HIV programmes to ensure 
Table 5 Factors associated with HIV infection in multivariate logistic regression model

\begin{tabular}{|c|c|c|}
\hline Variables in the final model & Adjusted OR $(95 \% \mathrm{Cl})$ & $p$ Value \\
\hline \multicolumn{3}{|l|}{ Community type } \\
\hline Rural & Reference & \\
\hline Urban & 2.7 (1.1 to 6.5$)$ & 0.03 \\
\hline \multicolumn{3}{|l|}{ Formal education attained } \\
\hline Primary (0-6years) & 1.7 (1.1 to 2.9$)$ & 0.04 \\
\hline High school or higher $(\geq 7)$ & Reference & \\
\hline \multicolumn{3}{|l|}{ Age (years) } \\
\hline$<25$ & Reference & \\
\hline $25-34$ & 2.1 (1.2 to 3.6$)$ & 0.01 \\
\hline$\geq 35$ & $2.6(1.3$ to 5.4$)$ & 0.01 \\
\hline \multicolumn{3}{|c|}{ Frequency of express and/or dressing as a woman } \\
\hline Not all the time & Reference & \\
\hline All the time & 2.1 (1.2 to 3.8$)$ & 0.01 \\
\hline \multicolumn{3}{|l|}{ Ever self-injected hormone } \\
\hline No & Reference & \\
\hline Yes & 4.4 (1.1 to 17.3$)$ & 0.03 \\
\hline \multicolumn{3}{|c|}{ Ulcerations or sores in the genital area in the past 12 months } \\
\hline No & Reference & \\
\hline Yes & 3.0 (1.2 to 7.8$)$ & 0.02 \\
\hline \multicolumn{3}{|c|}{ Used online services developed for men who have sex with men/transgender in the past sixmonths (eg, Facebook, website) } \\
\hline No & $1.9(1.2$ to 3.2$)$ & 0.01 \\
\hline Yes & Reference & \\
\hline
\end{tabular}

*Variables in the table were the ones that remained statistically significant after several steps of model fitting.

that poorly educated transgender women are reached with education, information, communication and skills related to HIV prevention and other health-related services.

Fifth, transgender women with self-reported STI symptoms (having ulcerations or sores in the genital area in the past 12 months) had a greater HIV prevalence. This is in line with existing evidence showing that STIs promote HIV transmission via a variety of biological mechanisms. ${ }^{40}$ Transgender women with high rates of STIs, particularly ulcerative genital diseases, are at high risk of HIV acquisition. $^{23}$

Finally, transgender women who reported never using online services developed for transgender women or MSM had an increased risk of HIV infection. Low use of online services by key populations in Cambodia is iterated in other studies. ${ }^{41}$ Non-users of online services, which tend to provide HIV information, education and communication, might have riskier sexual behaviours. Our data suggest that transgender women who did not use online services had higher numbers of overall male sexual partners (10.2 vs 6.3, $\mathrm{p}=0.002)$ and male commercial sexual partners $(0.8$ vs $0.6, \mathrm{p}=0.001)$ in the past three months. Also, they had a higher rate of inconsistent condom use with male commercial sexual partners in the past threemonths $(48.5 \%$ vs $34.4 \%, \mathrm{p}=0.004)$.
Unfortunately, in this study, we did not collect the details about the type of the online services, which could refer to dating, health and social services, or another type of online service. Studies in other settings confirm that transgender women with multiple sexual partners are exposed to a riskier level of HIV infection. ${ }^{6}{ }^{15}$ This finding suggests that access to community-based services tailored to transgender population can reduce their vulnerability to HIV as demonstrated in other settings. ${ }^{42}$ Sustaining and increasing the coverage of internet and peer-led community-based services for this population is particularly important, given that they are often unable to access health services due to stigmatisation and discrimination, ${ }^{161}$ even in healthcare settings. ${ }^{18}$ As in other settings globally, ${ }^{9}$ only half of those who were infected with HIV were aware of their status, demonstrating the need to intensify HIV testing, including self-testing at the community level, which transgender women in Cambodia are willing to use. ${ }^{43}$ Our study also found that once diagnosed the coverage of antiretroviral therapy was high in this population.

\section{LIMITATIONS OF THE STUDY}

Our study covered only the capital city and 12 provinces, which contain the highest numbers of transgender 
women. Since cities and provinces with fewer transgender women were left out, these results may not be generalised to all transgender women nationally. Second, the initial participant seeds were identified and recruited by outreach workers of community-based organisations, which could introduce bias towards transgender women under their programmes, leading to a recruitment bias. This problem could be exacerbated by outreach workers interviewing some participants who had received services from their NGO, which could have induced the participants' responses. Third, this study employed a self-reporting questionnaire on sensitive health and sexual behaviours, which may have been limited by social desirability bias. Fourth, although minimal, the monetary incentive given to the participants to recruit seeds may have affected their genuine motivation to partake in the study, which could influence their responses. Fifth, we used the backward selection of variables in the multivariable analysis. By using this method, we possibly dropped one or more variables that could be significant if we kept them until the final model. Finally, as this study was cross-sectional, it reports associations at a given time and may not be construed to be reporting causal relationships.

\section{CONCLUSIONS}

This study demonstrates that HIV prevalence among transgender women in Cambodia remains persistently high. With the prevalence rate at $5.9 \%$, higher prevalence was observed among transgender women who resided in urban areas, of older age, with low education levels, with previous genital sores, with a history of self-injected hormone and those who had never used online services developed for transgender women or MSM. Therefore, to eliminate new HIV infections among transgender women, tailored interventions need to focus on these subpopulations and attendant risk factors.

\section{Author affiliations}

${ }^{1}$ FHI 360, Phnom Penh, Cambodia

${ }^{2}$ KHANA Center for Population Health Research, Phnom Penh, Cambodia

${ }^{3}$ Population Services Khmer (PSK), Phnom Penh, Cambodia

${ }^{4}$ National Center for HIV/AIDS, Dermatology and STD (NCHADS), Phnom Penh,

Cambodia

${ }^{5}$ Asia Pacific Regional Office, FHI 360, Phnom Penh, Cambodia

${ }^{6}$ Division of Health Research, Lancaster University, Lancaster, UK

${ }^{7}$ Center for Global Health Research, Touro University California, Vallejo, CA, United States

Acknowledgements This study was conducted in collaboration between the consortium partners of the HIV/AIDS Flagship Project including KHANA, FHI360, PSI/ PSK and the 13 Municipal and Provincial Health Departments under the leadership of National Center for HIV/AIDS, Dermatology and STD (NCHADS). The authors thank all implementing partners, data collection teams and participants in the study who fully supported during the study design and data collection.

Contributors SC, ST, PM, CL, PC and SY designed the study, developed the research protocol and tools. SC, CN and SY analysed the data, interpreted the results and wrote the manuscript. PC, SC, CL, KP, ST and PM were responsible for training and data collection. JM, JD and GB supported findings analyses and manuscript writing. All authors read and approved the final manuscript.
Funding This study was conducted as part of the HIV/AIDS Flagship Project funded by President's Emergency Plan For AIDS Relief (PEPFAR) through the United States Agency for International Development (USAID).

Disclaimer Content of this paper is the responsibility of the authors and does not reflect the view of USAID or our respective institutions.

Competing interests None declared.

Ethics approval National Ethics Committee for Health Research (NECHR) of the Ministry of Health, Cambodia (no. 420 NECHR) and FHI 360's Protection of Human Subjects Committee (PHSC no. 713897).

Provenance and peer review Not commissioned; externally peer reviewed.

Data sharing statement Data used for this study cannot be made available in the manuscript, the supplemental files, or a public repository due to the ethical restriction stated in the agreement with the ethical committees. However, they can be accessed upon request from the principal investigator (SY) at siyan@doctor.com.

Open Access This is an Open Access article distributed in accordance with the Creative Commons Attribution Non Commercial (CC BY-NC 4.0) license, which permits others to distribute, remix, adapt, build upon this work non-commercially, and license their derivative works on different terms, provided the original work is properly cited and the use is non-commercial. See: http://creativecommons.org/ licenses/by-nc/4.0/

(c) Article author(s) (or their employer(s) unless otherwise stated in the text of the article) 2017. All rights reserved. No commercial use is permitted unless otherwise expressly granted.

\section{REFERENCES}

1. Baral SD, Poteat T, Strömdahl S, et al. Worldwide burden of HIV in transgender women: a systematic review and meta-analysis. Lancet Infect Dis 2013;13:214-22.

2. Silva-Santisteban A, Raymond HF, Salazar X, et al. Understanding the HIV/AIDS epidemic in transgender women of Lima, Peru: results from a sero-epidemiologic study using respondent driven sampling. AIDS Behav 2012;16:872-81.

3. Guadamuz TE, Wimonsate W, Varangrat A, et al. HIV prevalence, risk behavior, hormone use and surgical history among transgender persons in Thailand. AIDS Behav 2011;15:650-8.

4. Brennan J, Kuhns LM, Johnson AK, et al. Adolescent Medicine Trials Network for HIV/AIDS Interventions. Syndemic theory and HIVrelated risk among young transgender women: the role of multiple, co-occurring health problems and social marginalization. Am J Public Health 2012;102:1751-7.

5. Nemoto T, Operario D, Keatley J, et al. HIV risk behaviors among male-to-female transgender persons of color in San Francisco. Am J Public Health 2004;94:1193-9.

6. Clements-Nolle K, Marx R, Guzman R, et al. HIV prevalence, risk behaviors, health care use, and mental health status of transgender persons: implications for public health intervention. Am J Public Health 2001;91:915-21.

7. Gay and Lesbian Medical Association. Healthy people 2010 Companion Document for Lesbian, gay, bisexual, and transgender (LGBT) Health. San Francisco, CA, United States: Gay and Lesbian Medical Association, 2001.

8. Poteat T, Reisner SL, Radix A. HIV epidemics among transgender women. Curr Opin HIV AIDS 2014;9:168-73.

9. United Nations Programme on HIV/AIDS (UNAIDS). Fast Track: Ending the AIDS Epidemic By 2030. Geneva, Switzerland: UNAIDS, 2014.

10. Wiessing LG, van Roosmalen MS, Koedijk P, et al. Silicones, hormones and HIV in transgender street prostitutes. AIDS 1999;13:2315-6.

11. Global Forum on MSM and HIV. MSM and HIVIAIDS Risk in Asia: What Is Fueling the Epidemic Among MSM and How Can It Be Stopped? Oakland, CA, United States: Global Forum on MSM and HIV, 2006.

12. De Santis JP. HIV infection risk factors among male-to-female transgender persons: a review of the literature. J Assoc Nurses AIDS Care 2009;20:362-72.

13. Operario D, Nemoto T, Iwamoto M, et al. Unprotected sexual behavior and HIV risk in the context of primary partnerships for transgender women. AIDS Behav 2011;15:674-82.

14. Bockting WO, Robinson BE, Forberg J, et al. Evaluation of a sexual health approach to reducing HIV/STD risk in the transgender community. AIDS Care 2005;17:289-303. 
15. Bockting WO, Robinson BE, Rosser BR. Transgender HIV prevention: a qualitative needs assessment. AIDS Care 1998;10:505-25.

16. Chakrapani V, Newman PA, Shunmugam M, et al. Syndemics of depression, alcohol use, and victimisation, and their association with HIV-related sexual risk among men who have sex with men and transgender women in India. Glob Public Health 2017;12:250-65.

17. Nemoto T, Iwamoto M, Perngparn U, et al. HIV-related risk behaviors among kathoey (male-to-female transgender) sex workers in Bangkok, Thailand. AIDS Care 2012;24:210-9.

18. Scheim Al, Santos GM, Arreola S, et al. Inequities in access to HIV prevention services for transgender men: results of a global survey of men who have sex with men. J Int AIDS Soc 2016;19(3 Suppl 2):20779.

19. Schneiders ML, Weissman A. Determining barriers to creating an enabling environment in Cambodia: results from a baseline study with key populations and police. J Int AIDS Soc 2016;19(4 Suppl 3):20878.

20. Brennan J, Kuhns LM, Johnson AK, et al. Syndemic theory and HIVrelated risk among young transgender women: the role of multiple, co-occurring health problems and social marginalization. Am J Public Health 2012;102:1751-7.

21. Raiford JL, Hall GJ, Taylor RD, et al. The role of structural barriers in Risky Sexual Behavior, victimization and readiness to Change HIV/ STI-Related risk Behavior among Transgender Women. AIDS Behav 2016;20:2212-21.

22. Weissman A, Ngak S, Srean C, et al. HIV prevalence and risks associated with HIV infection among transgender individuals in Cambodia. PLoS One 2016;11:e0152906.

23. Chhorvann $\mathrm{C}$, Vonthanak S. Estimations and projections of HIV/AIDS in Cambodia 2010-2015. Phnom Penh, Cambodia: National Center for HIVIAIDS, Dermatology and STDs 2011.

24. Liu K, Chhea C. The BROS Khmer: behavioral risks on-site serosurvey among at-risk urban men in Cambodia. . Phnom Penh, Cambodia: FHI, 2010.

25. National Center for HIVIAIDS, Dermatology and STD (NCHADS) Standard Operation Procedure (SoP) for Boosted Continuum of Prevention to Care and treatment for most at risk Population in Cambodia. Phnom Penh, Cambodia: NCHADS, 2013.

26. Mun P, Chhim S, Chhoun P, et alNational Popualtion size estimation, HIV related risk Behaoviors, and HIV prevlence among men who sex with men in Cambodia in 2014Phnom Penh, Cambodia NCHADS/ KHANA 2016.

27. Size estimation of Transgender Population in Cambodia.Phnom Penh, Cambodia: FHI, 2012.

28. National Center for HIVIAIDS, detamatology and STD (NCHADS). Standard operating procedures for HIV testing and counseling (HTC). Phnom Penh, Cambodia: NCHADS, 2012.

29. Behavioral Surveilance surveys (BSS). Guideline for Repeated behavioral Surveys in Population at Risk of HIV. Washington DC, United States: FHI, 2000.
30. Mun P, Sopheab H, Tuot S, et al. National HIV sentinel survey among women attending antenatal care in Cambodia 2014. Phnom Penh, Cambodia NCHADS 2016

31. National Center for HIVIAIDS, Dermatology and STDs (NCHADS). Cambodia Sub-National HIV estimates and Projection. Phnom Penh, Cambodia: NCHADS, 2016.

32. Yi S, Chhim S, Chhoun P, et al. Men who have sex with men in Cambodia: population size, HIV risky behaviors, and HIV prevalence. Am J Epidemiol Infect Dis 2016;4:91-9.

33. Nuttbrock L, Hwahng S, Bockting W, et al. Lifetime risk factors for HIV/sexually transmitted infections among maleto-female transgender persons. J Acquir Immune Defic Syndr 2009;52:417-21.

34. Melendez RM, Pinto R. 'It's really a hard life': love, gender and HIV risk among male-to-female transgender persons. Cult Health Sex 2007;9:233-45.

35. Sevelius JM. Gender Affirmation: A Framework for Conceptualizing Risk Behavior among Transgender Women of Color. Sex Roles 2013;68:675-89.

36. Sausa LA, Keatley J, Operario D. Perceived risks and benefits of sex work among transgender women of color in San Francisco. Arch Sex Behav 2007;36:768-77.

37. Yi S, Ngin C, Tuot S, et al. HIV prevalence, risky behaviors, and discrimination experiences among transgender women in Cambodia:Descriptive findings from a 651 national integrated biological and behavioral survey: BMC Int Health Hum Rights. In Press, 2016.

38. Sanchez NF, Sanchez JP, Danoff A, et al. Health care utilization, barriers to care, and hormone usage among male-to-female transgender persons in New York City. Am J Public Health 2009;99:713-9.

39. Simon PA, Reback CJ, Bemis CC, et al. HIV prevalence and incidence among male-to-female transsexuals receiving HIV prevention services in Los Angeles County. AIDS 2000;14:2953-5.

40. Fleming DT, Wasserheit JN. From epidemiological synergy to public health policy and practice: the contribution of other sexually transmitted diseases to sexual transmission of HIV infection. Sex Transm Infect 1999;75:3-17.

41. Innovate HIV, Project E. University Research Co. LLC (URC-HIEP). evaluation of the HIV program for Transgender Women in Cambodia. Phnom Penh, Cambodia: URC-HIEP, 2016.

42. Shaikh S, Mburu G, Arumugam V, et al. Empowering communities and strengthening systems to improve transgender health: outcomes from the Pehchan programme in India. J Int AIDS Soc 2016;19(3 Suppl 2):20809.

43. Pal K, Ngin C, Tuot S, et al. Acceptability Study on HIV Self-Testing among Transgender Women, Men who Have Sex with Men, and Female Entertainment Workers in Cambodia: A Qualitative Analysis. PLoS One 2016;11:e0166129. 